\title{
Probing the neutrino mass matrix in next generation neutrino oscillation experiments
}

\author{
Sandhya Choubey ${ }^{1, *}$ \\ 1 Rudolf Peierls Centre for Theoretical Physics, \\ University of Oxford, 1 Keble Road, Oxford OX1 3NP, UK
}

\begin{abstract}
We review the current status of the neutrino mass and mixing parameters needed to reconstruct the neutrino mass matrix. A comparative study of the precision in the measurement of oscillation parameters expected from the next generation solar, atmospheric, reactor and accelerator based experiments is presented. We discuss the potential of $0 \nu \beta \beta$ experiments in determining the neutrino mass hierarchy and the importance of a better $\theta_{12}$ measurement for it.
\end{abstract}

\section{INTRODUCTION}

The last few years have provided us with conclusive proof of the existence of oscillations and hence mass and mixing in the neutrino sector. While the atmospheric neutrino data from Super-Kamiokande (SK) and the accelerator data from the K2K have confirmed oscillations in the $\nu_{\mu}-\nu_{\tau}$ sector with best-fit $\Delta m_{31}^{2}=2.1 \times 10^{-3} \mathrm{eV}^{2}$ and $\sin ^{2} 2 \theta_{23}=1$ [1] , the combined data from the solar neutrino experiments and the latest spectacular results from the KamLAND reactor experiment can be explained only by oscillations of $\nu_{e}\left(\bar{\nu}_{e}\right)$ with best-fit $\Delta m_{21}^{2}=8.0 \times 10^{-5} \mathrm{eV}^{2}$ and $\sin ^{2} \theta_{12}=0.31[2]$. Thus, having established the existence of neutrino mass and mixing, these results have proclaimed a new era in Neutrino Physics, where the emphasis has shifted from unveiling the reasons for solar/atmospheric neutrino deficit to making increasingly precise measurements of neutrino oscillation parameters, a prerequisite for any progress in our understanding of the origin of the patterns of solar and atmospheric neutrino mass and mixing.

In the present article we discuss the possibilities of high precision measurement of the solar and atmospheric neutrino oscillation parameters with future data from solar, reactor,

*Electronic address: sandhya@thphys.ox.ac.uk 
atmospheric and long baseline neutrino experiments. We expound the possibility of measuring the deviation of $\theta_{23}$ from maximality using earth matter effects in atmospheric neutrinos. Finally we study the feasibility of using $0 \nu \beta \beta$ experiments to determine the neutrino mass hierarchy and point the necessity for better $\sin ^{2} \theta_{12}$ measurements for accomplishing it.

\section{SOLAR NEUTRINO OSCILLATION PARAMETERS}

\section{A. Bounds from Current Solar and Reactor Experiments}

\begin{tabular}{|c|c|c|c|c|}
\hline Data set used & Range of $\Delta m_{21}^{2}$ & Spread in $\Delta m_{21}^{2}$ & $\sin ^{2} \theta_{12}$ & Spread in $\sin ^{2} \theta_{12}$ \\
\hline only solar & $(3.3-18.4) \times 10^{-5} \mathrm{eV}^{2}$ & $69 \%$ & $0.24-0.41$ & $26 \%$ \\
\hline solar + 766.3 Ty KL & $(7.2-9.2) \times 10^{-5} \mathrm{eV}^{2}$ & $12 \%$ & $0.25-0.39$ & $22 \%$ \\
\hline solar(SNO3) + 766.3 Ty KL & $(7.2-9.2) \times 10^{-5} \mathrm{eV}^{2}$ & $12 \%$ & $0.26-0.37$ & $18 \%$ \\
\hline solar(SNO3) + 3KTy KL & $(7.6-8.6) \times 10^{-5} \mathrm{eV}^{2}$ & $6 \%$ & $0.26-0.36$ & $16 \%$ \\
\hline
\end{tabular}

TABLE I: The $3 \sigma$ allowed ranges and $\%$ spread of $\Delta m_{21}^{2}$ and $\sin ^{2} \theta_{12}$ from a 1 parameter fit.

We present in Table [3] the $3 \sigma$ allowed range of $\Delta m_{21}^{2}$ and $\sin ^{2} \theta_{12}$ that we have from the current available solar and reactor neutrino data. We also show the corresponding "spread"

$$
\text { spread }=\frac{\text { prm }_{\max }-\mathrm{prm}_{\min }}{\text { prm }_{\max }+\text { prm }_{\min }} \times 100 .
$$

Sensitivity of KamLAND to the shape and hence distortion of the reactor $\bar{\nu}_{e}$ induced positron spectrum, gives the experiment a tremendous ability to constrain $\Delta m_{21}^{2}$. However, we can see from Table \that it is not much sensitive to the mixing angle $\theta_{12}$. The average energy and distance in KamLAND corresponds to $\sin ^{2}\left(\Delta m_{21}^{2} L / 4 E\right) \approx 0$, i.e, it is situated close to a Survival Probability MAXimum (SPMAX). This means that the coefficient of the $\sin ^{2} 2 \theta_{12}$ term in $P_{\bar{e} \bar{e}}^{K L}$ is relatively small, smothering the sensitivity of KamLAND to $\theta_{12}$ [4, 5].

Also given in Table \are the expected bounds on $\Delta m_{21}^{2}$ and $\sin ^{2} \theta_{12}$ after the prospective future data from the running SNO and KamLAND experiments become available. For SNO we assume that the third and final phase of the experiment will measure the same Neutral Current (NC) and Charged Current (CC) rates as the salt phase, but with reduced errors of $6 \%$ and $5 \%$ respectively. For KamLAND we simulate the $3 \mathrm{kTy}$ data at $\Delta m_{21}^{2}=8.0 \times 10^{-5}$ $\mathrm{eV}^{2}$ and $\sin ^{2} \theta_{12}=0.3$ and use a systematic error of $5 \%$. The uncertainty in $\Delta m_{21}^{2}$ is expected 

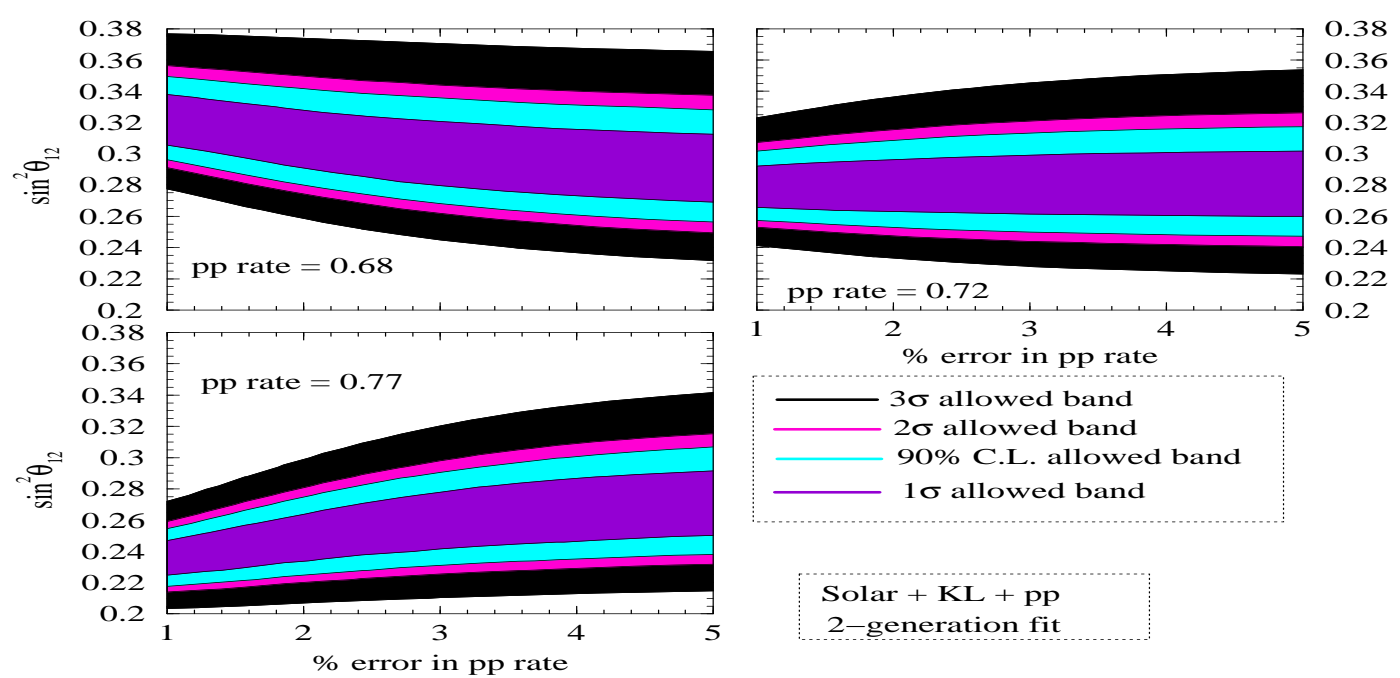

FIG. 1: Sensitivity plot showing the allowed range of $\sin ^{2} \theta_{12}$ as a function of the error in $p p$ rate for three different values of measured $p p$ rate.

to reduce to $6 \%$ with $3 \mathrm{kTy}$ data from KamLAND. The uncertainty in $\sin ^{2} \theta_{12}$ is expected to improve after the phase-III results from SNO to $18 \%$. This would improve to about $16 \%$ if the SNO phase-III projected results are combined with the $3 \mathrm{kTy}$ simulated data from KamLAND. However, we note that even with the combined data from phase-III of SNO and 3 kTy statistics from KamLAND, the uncertainty on $\sin ^{2} \theta_{12}$ would stay well above the $10-15 \%$ level at $3 \sigma$.

\section{B. Precision Expected from Next Generation Experiments}

In this sub-section we explore the possibility of new solar and reactor neutrino experiments and make a comparative study of their sensitivity to $\Delta m_{21}^{2}$ and $\sin ^{2} \theta_{12}$.

The Generic $p p$ experiment: Sub-MeV solar neutrino experiments (LowNu experiments) are being planned for detecting the $p p$ neutrinos using either charged current reactions (LENS, MOON, SIREN) or electron scattering process (XMASS, CLEAN, HERON,

MUNU, GENIUS) 6]. It has been realized that high precision measurement of the $p p$ neutrino flux can be instrumental for more accurate determination of the neutrino mixing parameter, which as we have seen in the earlier section, will not be determined to an accuracy of below $10-15 \%$ by the current set of experiments. 


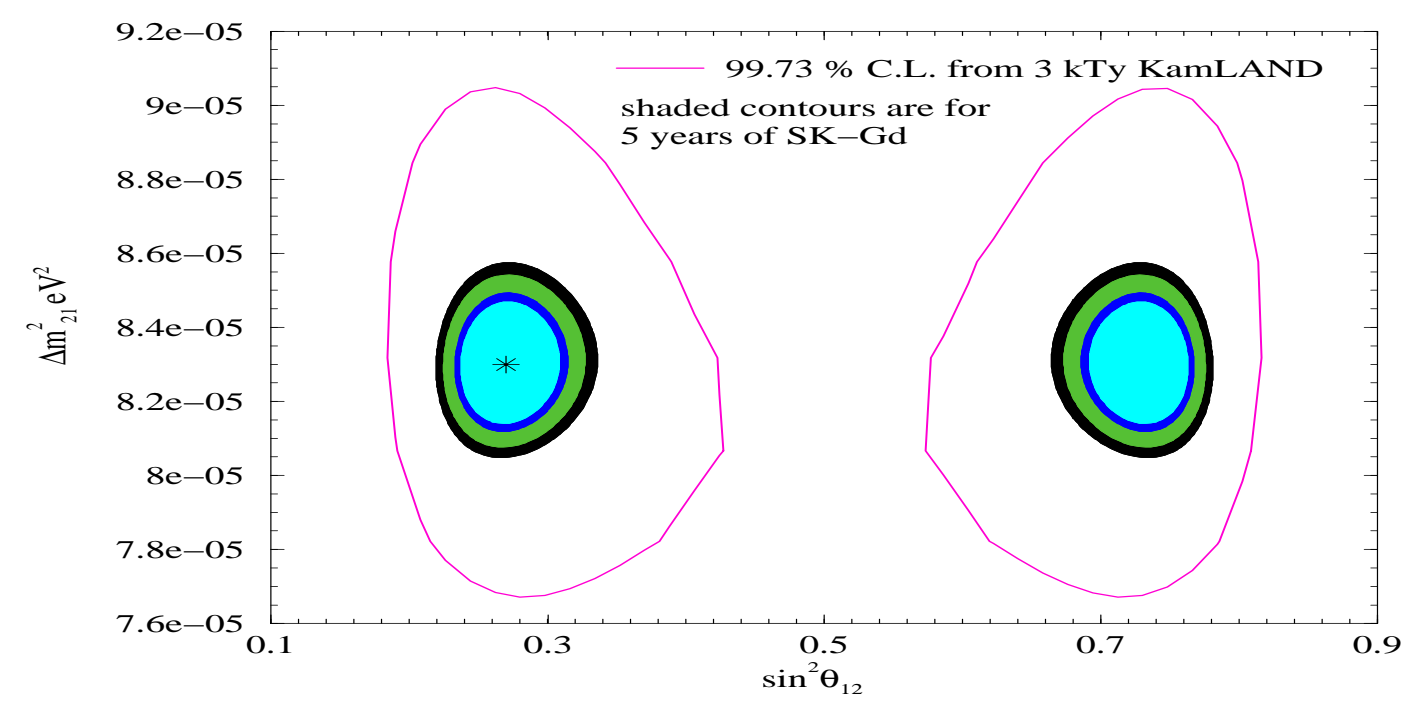

FIG. 2: The $90 \%, 95 \%, 99 \%, 99.73 \%$ C.L. allowed regions in the $\Delta m_{21}^{2}-\sin ^{2} \theta_{12}$ plane from an analysis of prospective data, obtained in 5 years of running of the SK-Gd detector. The open contours shows the $99.73 \%$ C.L. allowed areas expected from 3 kTy of KamLAND data.

We consider a generic $\nu_{e}-e$ scattering experiment with a threshold of $50 \mathrm{keV}$. This experiment will be sensitive to the $p p$ neutrinos. In Fig. [ we plot the two-generation allowed range of $\sin ^{2} \theta_{12}$ from the global analysis of KamLAND and solar data including the LowNu $p p$ rate, as a function of the error in the $p p$ measurement. We consider three illustrative $p p$ rates of $0.68,0.72$ and 0.77 and vary the experimental error in the $p p$ measurement from 1 to $5 \%$. By adding the $p p$ flux data in the analysis, the error in $\sin ^{2} \theta_{12}$ determination reduces to $14 \%(19 \%)$ at $3 \sigma$ for $1 \%(3 \%)$ uncertainty in the measured $p p$ rate. Performing a similar three-neutrino oscillation analysis we have found that, as a consequence of the uncertainty on $\sin ^{2} \theta_{13}$, the error on the value of $\sin ^{2} \theta_{12}$ increases correspondingly to $17 \%(21 \%)$ [7].

The SK-Gd reactor experiment: There has been a proposal to dope SuperKamiokande (SK) with Gd by dissolving $0.2 \%$ of gadolinium chloride in the SK water [8]. SK gets the same reactor flux as KamLAND, and in principle could detect these reactor $\bar{\nu}_{e}$ through their capture on protons, which releases a positron and a neutron. The detector has to tag these neutrons through delayed coincidence to be able to unambiguously observe the reactor $\bar{\nu}_{e}$. However, neutron capture on proton releases a $2.2 \mathrm{MeV} \gamma$, which is undetectable in SK. Addition of Gadolinium in SK would circumvent this problem since neutron capture on gadolinium releases a $8 \mathrm{MeV} \gamma$ cascade, which is above the SK threshold and 


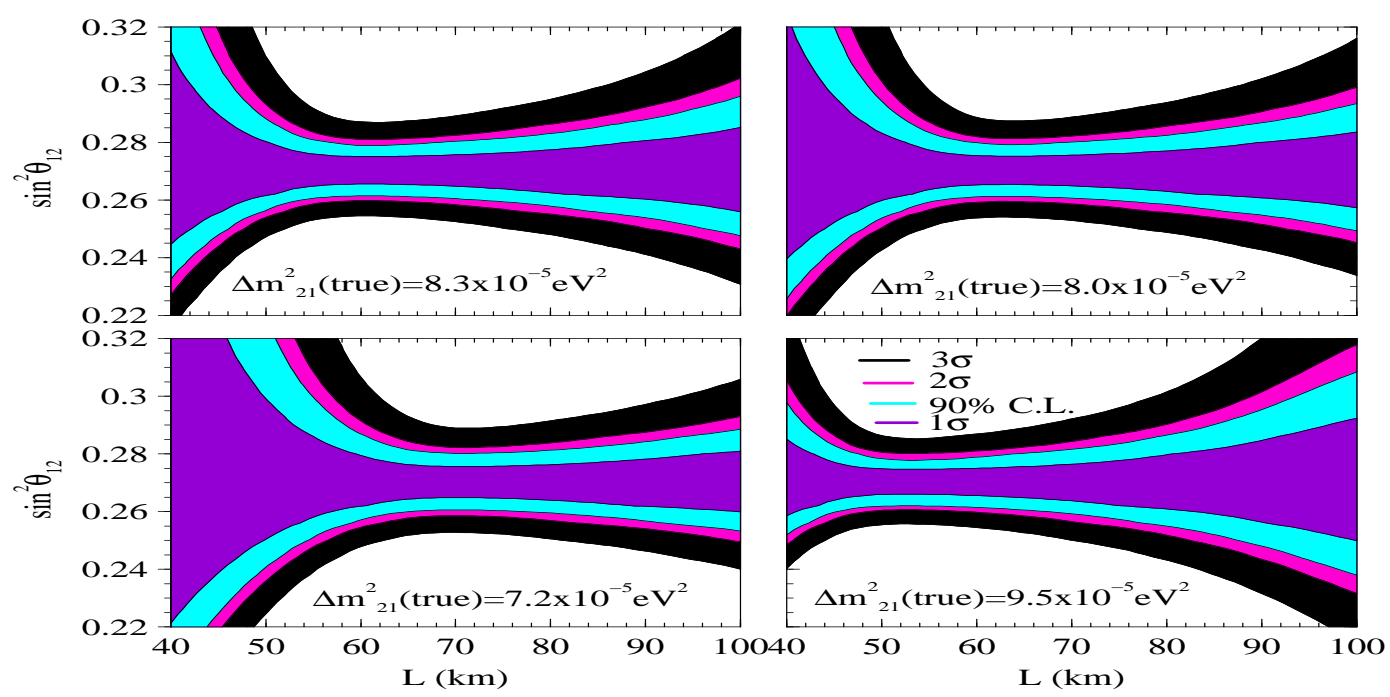

FIG. 3: Sensitivity plots for the SPMIN reactor experiment showing the $1 \sigma, 1.64 \sigma, 2 \sigma$, and $3 \sigma$ range of allowed values for $\sin ^{2} \theta_{12}$ as a function of the baseline $L$.

hence possible to observe. With its 22.5 kton of ultra pure water, the SK detector has about $1.5 \times 10^{33}$ free protons as target for the antineutrinos coming from various reactors in Japan. Therefore for the same measurement period, the SK-Gd reactor experiment is expected to have about 43 times the statistics of the KamLAND experiment.

We simulate the reactor $\bar{\nu}_{e}$ data expected in the proposed SK-Gd detector at $\Delta m_{21}^{2}=$ $8.3 \times 10^{-5} \mathrm{eV}^{2}$ and $\sin ^{2} \theta_{12}=0.27$ and divide it into 18 energy bins, with a visible energy threshold of $3 \mathrm{MeV}$ and bin width of $0.5 \mathrm{MeV}$. The results of the statistical analysis of this prospective data is presented in Fig. 2 for 5 years of exposure. Also shown in the figure for comparison is the $99.73 \%$ C.L. line expected from a $3 \mathrm{kTy}$ prospective data in KamLAND. We can clearly see that the precision expected in both $\Delta m_{21}^{2}$ and $\sin ^{2} \theta_{12}$ is much better in SK-Gd. The spread in $\Delta m_{21}^{2}$ and $\sin ^{2} \theta_{12}$ expected from 5 years of data in SK-Gd would be at the level of $2-3 \%$ and $18 \%$ respectively at $3 \sigma$. This can be compared with the corresponding spread of $6 \%$ and $32 \%$ expected from $3 \mathrm{kTy}$ of KamLAND data.

The SPMIN reactor experiment: The solar mixing angle could be measured with unprecedented accuracy in a reactor experiment with the baseline tuned to the Survival Probability MINimum (SPMIN). This idea was proposed in [4], where the optimal baseline for the most accurate measurement of $\sin ^{2} \theta_{12}$ for the old low-LMA best-fit $\Delta m_{21}^{2}=7.2 \times 10^{-5}$ eV2 was found to be $70 \mathrm{~km}$ from a statistical analysis. 
In Fig. 3 we show the $\sin ^{2} \theta_{12}$ sensitivity expected in a reactor experiment as a function of the baseline $L$. We assume a total systematic uncertainty of $2 \%$ and consider a statistics of $73 \mathrm{GWkTy}$ (given as a product of reactor power in GW and the exposure of the detector in $\mathrm{kTy}$ ). We assume that the true value of $\sin ^{2} \theta_{12}=0.27$ and simulate the prospective observed positron spectrum in the detector for four different assumed true values of $\Delta m_{21}^{2}$. We simulate the spectrum at each baseline and plot the range of values of $\sin ^{2} \theta_{12}$ allowed by the experiment as a function of the baseline. The baseline at which the band of allowed values of $\sin ^{2} \theta_{12}$ is most narrow is the ideal baseline for the SPMIN reactor experiment. The figure confirms that this ideal baseline depends crucially on the true value of $\Delta m_{21}^{2}$. The most optimal baseline for the true value of $\Delta m_{21}^{2}=8.3(8.0) \times 10^{-5} \mathrm{eV}^{2}$ is seen to be $60(63) \mathrm{km}$. At the most optimal baseline, the SPMIN reactor experiment can achieve an unprecedented accuracy of $\sim 2(6) \%$ at $1 \sigma(3 \sigma)$ in the measurement of $\sin ^{2} \theta_{12}$.

From the Fig. 3] we get an impression that the optimal baseline for a given true value of $\Delta m_{21}^{2}$ is very finely tuned. However, note that in Fig. 3 we had allowed $\Delta m_{21}^{2}$ to vary freely. The uncertainty in the $\Delta m_{21}^{2}$ measurement translates to extra uncertainty in the $\sin ^{2} \theta_{12}$ measurement. If $\Delta m_{21}^{2}$ could be fixed, assumed to be measured to a very high precision in some other experiment like KamLAND or SK-Gd, then the uncertainty in $\sin ^{2} \theta_{12}$ due to $\Delta m_{21}^{2}$ can be drastically reduced. If $\Delta m_{21}^{2}$ was kept fixed, then the choice of the baseline for setting up the SPMIN experiment becomes much broader 7]].

One of the driving features for this kind of a precision experiment is the statistics. We have checked that the sensitivity of $\sin ^{2} \theta_{12}$ improves from $3(10) \%$ to $2(6) \%$ at $1 \sigma(3 \sigma)$ as the statistics is increased from $20 \mathrm{GWkTy}$ to $60 \mathrm{GWkTy}$. Another important aspect is the systematic uncertainty. Fig. 3 has been generated with an assumed $2 \%$ systematic uncertainty, which could be experimentally very challenging. The effect of systematics on the $\sin ^{2} \theta_{12}$ measurement can be checked by repeating the analysis with a more conservative estimate of $5 \%$ for the systematic uncertainty. We find that for $\Delta m_{21}^{2}($ true $)=8.3 \times 10^{-5}$ $\mathrm{eV}^{2}$, the spread in $\sin ^{2} \theta_{12}$ at $L=60 \mathrm{~km}$ increases from $6.1 \%$ to $8.6 \%$ at $3 \sigma$, as the systematic error is increased from $2 \%$ to $5 \%$. Finally, the impact of the $\theta_{13}$ uncertainty on the precision of $\sin ^{2} \theta_{12}$ is to increase the uncertainty in $\sin ^{2} \theta_{12}$ from $6.1 \%$ to $8.7 \%$ at $3 \sigma$, for $\Delta m_{21}^{2}($ true) $=$ $8.3 \times 10^{-5} \mathrm{eV}^{2}$ and $L=60 \mathrm{~km}$. 


\section{ATMOSPHERIC NEUTRINO OSCILLATION PARAMETERS}

The parameters $\Delta m_{31}^{2}$ and $\sin ^{2} \theta_{23}$ at present are best constrained by the SK atmospheric neutrino data. The $3 \sigma$ allowed ranges (spread) of $\Delta m_{31}^{2}$ and $\sin ^{2} \theta_{23}$ obtained from the final analysis by the SK collaboration are $(1.3-4.2) \times 10^{-3} \mathrm{eV}^{2}(53 \%)$ and $0.33-0.66(34 \%)$ [1]. The addition of the $\mathrm{K} 2 \mathrm{~K}$ data sample into the analysis brings in a modest improvement in the range of $\Delta m_{31}^{2}$ to (1.4-3.3) $\times 10^{-3} \mathrm{eV}^{2}(42 \%)$, however the the uncertainty in the allowed range of $\sin ^{2} \theta_{23}$ remains the same [10].

Both $\Delta m_{31}^{2}$ and $\sin ^{2} 2 \theta_{23}$ are expected to be measured very accurately by the forthcoming long baseline (LBL) experiments. A statistical analysis of the combined data set with five years of running of MINOS, ICARUS, OPERA, T2K and NO $\nu \mathrm{A}$ each, reveals that $\Delta m_{31}^{2}$ and $\sin ^{2} \theta_{23}$ could be measured with a spread of $4.5 \%$ and $20 \%$ respectively at $3 \sigma$ [1]. The future prospective data from water Cerenkov atmospheric neutrino experiments with a statistics 20 times the current SK statistics could measure $\Delta m_{31}^{2}$ and $\sin ^{2} \theta_{23}$ with a spread of $\sim 17 \%$ and $\sim 24 \%$ respectively 12 . A large magnetized iron calorimetric detector such as the proposed INO detector ICAL [13], could use atmospheric neutrinos to measure $\Delta m_{31}^{2}$ and $\sin ^{2} \theta_{23}$ with an accuracy comparable to that expected from the combined data from the LBL experiments [14].

\section{A. Is the Mixing Angle $\theta_{23}$ Maximal?}

The measurement of both the magnitude and sign of the deviation of $\sin ^{2} \theta_{23}$ from its maximal value 0.5 is of utmost theoretical importance. In this section we will see how well the next generation of experiments can answer the question: Is the mixing angle $\theta_{23}$ maximal? To quantify the deviation of the true value of $\theta_{23}$ from its maximal value, we introduce the function $D \equiv \frac{1}{2}-\sin ^{2} \theta_{23}$. The best current limit on $D$ comes from the SK atmospheric neutrino experiment giving $|D| \leq 0.16$ at $3 \sigma$ [1]. More precise measurements of $\theta_{23}$ are expected from future high statistics atmospheric neutrino data in water Cerenkov detectors. Better constraints are expected from forthcoming LBL experiments, owing to their larger statistics and lower systematic errors. However, in both these classes of experiments, $\theta_{23}$ will be mainly determined by the $\nu_{\mu}$ (and/or $\bar{\nu}_{\mu}$ ) disappearance channel in vacuum, which predominantly depends on $\sin ^{2} 2 \theta_{23}$. This leads to two very important consequences. Firstly, 


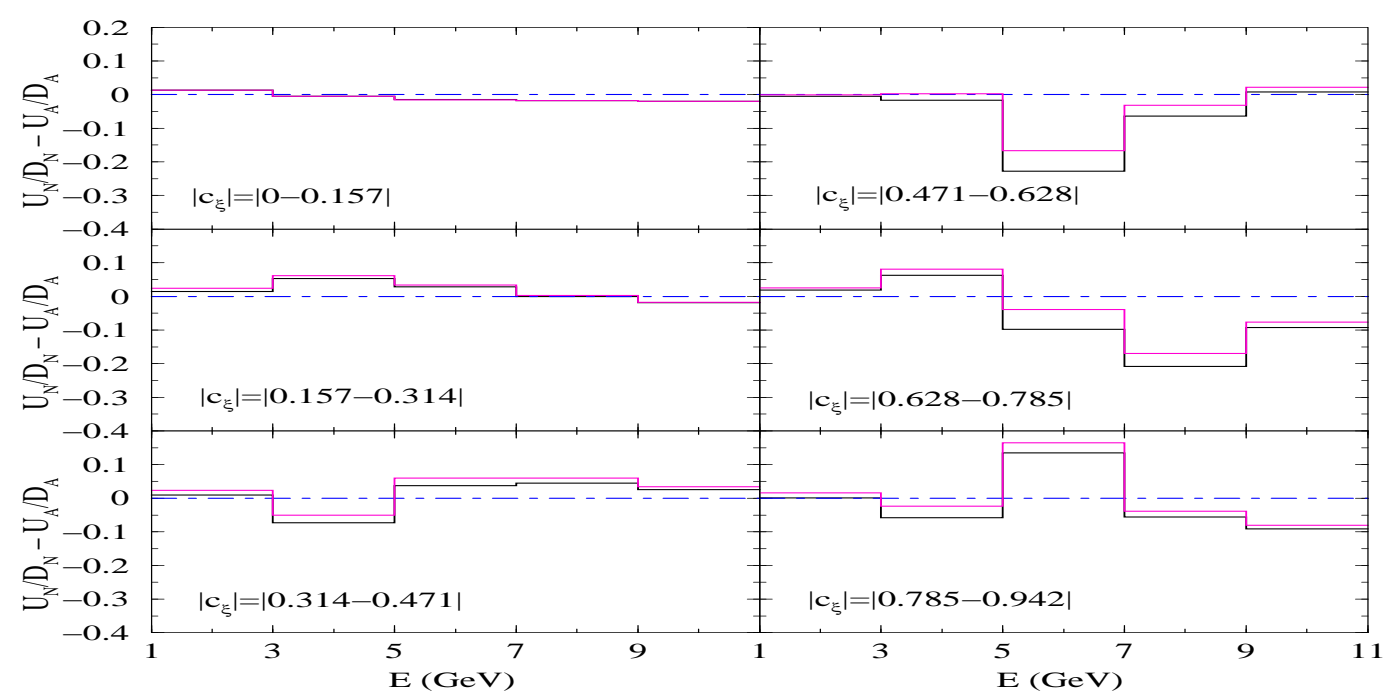

FIG. 4: The difference between the up-down ratio for the neutrinos $\left(U_{N} / D_{N}\right)$ and antineutrinos $\left(U_{A} / D_{A}\right)$ shown for the various energy and zenith angle bins. The solid black and solid magenta lines are for neutrinos/antineutrinos travelling in matter with $\sin ^{2} \theta_{23}=0.5$ and 0.36 respectively.

the fact that $\theta_{23}$ is close to maximal and $\delta\left(\sin ^{2} \theta_{23}\right)=\delta\left(\sin ^{2} 2 \theta_{23}\right) /\left(4 \cos 2 \theta_{23}\right)$ implies that even though one could determine the value of $\sin ^{2} 2 \theta_{23}$ at the percentage level from the next generation long baseline experiments, the uncertainty in $\sin ^{2} \theta_{23}$ would still remain in the region of $10-20 \%$, depending on the true value of $\sin ^{2} \theta_{23}\left(\sin ^{2} \theta_{23}\right.$ (true)). The second consequence of the predominant $\sin ^{2} 2 \theta_{23}$ dependence of $P_{\mu \mu}$ means that they are almost insensitive to the octant of $\theta_{23}$ and hence to the sign of $D$.

Large matter effects are known to exist in $P_{\mu \mu}$ for neutrinos travelling over very long distances with $L \sim 7000 \mathrm{~km}$. However, the net matter effects given by $\Delta P_{\mu \mu}\left(=P_{\mu \mu}-\right.$ $\left.P_{\mu \mu}^{v a c u u m}\right)$ changes sign with energy $E$ and baseline length $L$. Therefore, in order to maximize the observation of matter effects in the atmospheric neutrino disappearance probability, one needs a detector with very good energy and zenith angle $(\xi)$ resolution. Large magnetized iron calorimeter detectors like the one proposed for the ICAL detector at INO [13], allows for good $E$ and $L$ resolution and at the same time can distinguish the $\nu_{\mu}$ from the $\bar{\nu}_{\mu}$ signal, further enhancing the sensitivity to matter effects. Observation of such large matter effects in atmospheric neutrinos can be used for unambiguous determination of the neutrino mass hierarchy [16]. It has been quantitatively argued in 17] that presence of large matter effects 


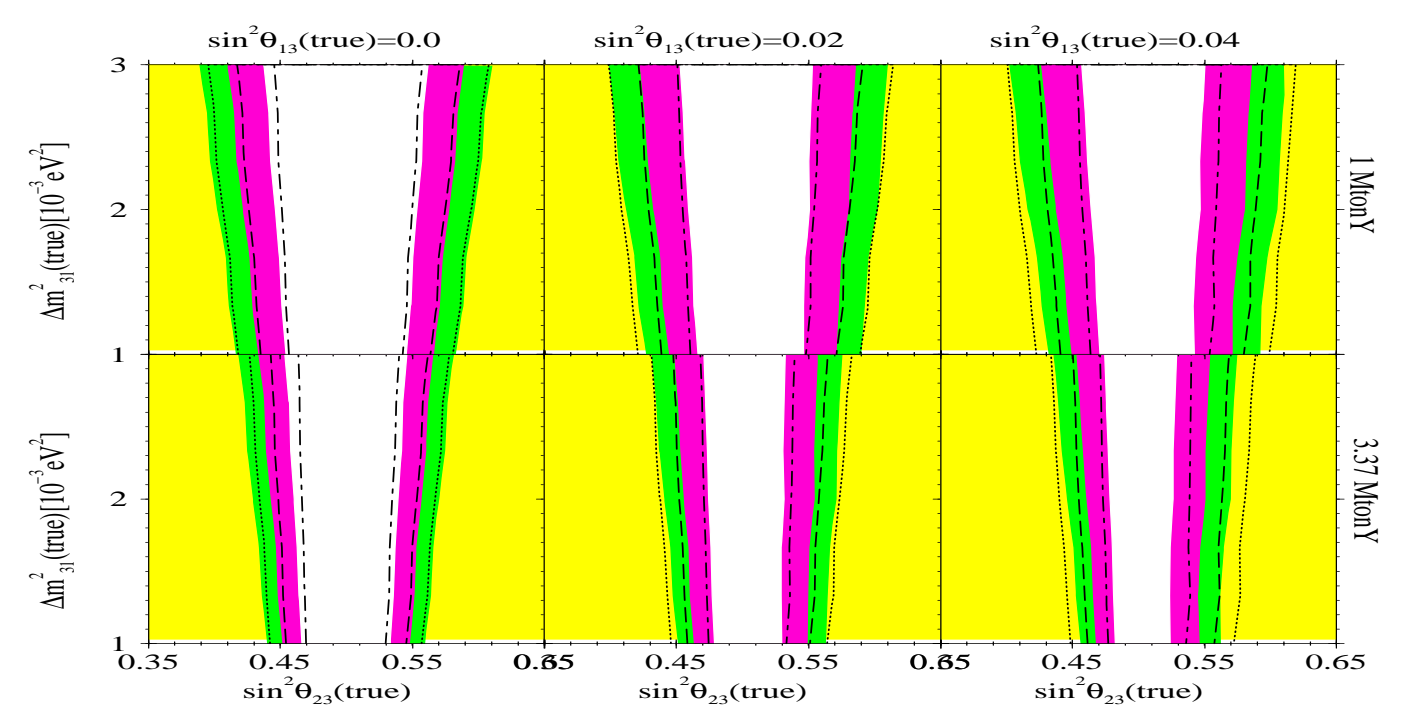

FIG. 5: The regions of $\Delta m_{31}^{2}$ (true) and $\sin ^{2} \theta_{23}$ (true) where maximal $\theta_{23}$ mixing can be rejected by using 1 MtonY (upper panels) and 3.37 MtonY (lower panels) atmospheric neutrino data in ICAL at $1 \sigma$ (white band), $2 \sigma$ (blue band) and $3 \sigma$ (green band). The hollow dark lines show the corresponding bands for neutrinos travelling in pure vacuum.

in $P_{\mu \mu}$ enhances its sensitivity to $\theta_{23}$ as well. ${ }^{1}$ Fig. 4 shows the impact of matter effects on atmospheric neutrinos events in an ICAL-like detector, for the normal mass hierarchy. The figure shows the difference in the up-down ratio $(U / D)$ of the $\nu_{\mu}(N)$ and $\bar{\nu}_{\mu}(A)$ events, and have been binned into five energy and 12 zenith angle bins. For instance, the first (last) panel on the top left (bottom right) contains $U_{N} / D_{N}-U_{A} / D_{A}$ for $0 \leq|\cos \xi| \leq 0.157$ $(0.785 \leq|\cos \xi| \leq 1)$ and calculated by taking upward going neutrinos with $-0.157 \leq$ $\cos \xi \leq 0(-1 \leq \cos \xi \leq-0.785)$ and downward going neutrinos with $0 \leq \cos \xi \leq 0.157$ $(0.785 \leq \cos \xi \leq 1)$. We note that matter effects are largest in the $E=5-7 \mathrm{GeV}$ and $-0.628 \leq \cos \xi \leq-0.471$ bin. We also note that reducing $\sin ^{2} \theta_{23}$ from the maximal 0.5 to 0.36 brings a nearly $10 \%$ change in the difference $U_{N} / D_{N}-U_{A} / D_{A}$.

We perform a statistical analysis of the simulated data sample in an ICAL-like detector, adding the constraint on $\sin ^{2} \theta_{13}$ expected from future reactor and accelerator experiments [1]. Our procedure is to generate the data at a certain nonmaximal value of $\sin ^{2} \theta_{23}$ (true) and then fit this data with the maximal $\sin ^{2} \theta_{23}=0.5$, choosing different val-

\footnotetext{
$\overline{{ }^{1} \text { See }[18]}$ for a discussion on using matter effects in $P_{\mu \mu}$ with $L \sim 1000 \mathrm{~km}$ to test maximality.
} 
ues of $\sin ^{2} \theta_{23}$ (true). The details of the statistical analysis can be found in [17]. The results are displayed in Fig. 5, where the upper and lower panels correspond to respective exposures of 1 MtonY and 3.37 MtonY. The regions of $\sin ^{2} \theta_{23}$ (true) and $\Delta m_{31}^{2}$ (true) within the white, blue and green bands of Fig. [5 show the true values of those quantities for which the distinction of a maximal from a true nonmaximal value of $\theta_{23}$ will not be possible at the $1 \sigma, 2 \sigma$ and $3 \sigma$ levels respectively for the specified exposure. The broken lines give the corresponding limits of $\sin ^{2} \theta_{23}$ (true) in case earth matter effects were deliberately switched off by hand. A comparison of the broken lines with the corresponding continuous lines show that matter effects tend to increase somewhat the sensitivity of ICAL to test the maximality of $\sin ^{2} \theta_{23}$. Specifically, for $\Delta m_{31}^{2}($ true $)=2.0 \times 10^{-3} \mathrm{eV}^{2}$ and $\sin ^{2} \theta_{13}$ (true) $=0.04(0.00), \sin ^{2} \theta_{23}($ true $)$ can be distinguished by ICAL from the maximal value of 0.5 at the $3 \sigma$ level within $17 \%$ (20\%) from 1 MtonY of exposure and within 11\% (14\%) if the statistics was increased to 3.37 MtonY. This is comparable to the sensitivity of the combined data from the forthcoming accelerator-based LBL experiments to a deviation from maximality of $\sin ^{2} \theta_{23}$, which is 15 ] $\sim 14 \%$ at $3 \sigma$ (for $\Delta m_{31}^{2}$ (true) $=2.5 \times 10^{-3} \mathrm{eV}^{2}$ ). Our sensitivity to $D$ is also comparable to that expected with atmospheric neutrinos in very large futuristic water Cerenkov detectors. For statistics that is 20 (50) times the current SK statistics, denoted as SK20 (SK50), a very large water Cerenkov atmospheric neutrino experiment is expected to test a deviation from a maximal $\sin ^{2} \theta_{23}$ upto [12] $23 \%(19 \%)$ at $3 \sigma$.

\section{B. Sensitivity to the octant of $\theta_{23}$}

If the true value of $\theta_{23}$ is not $45^{\circ}$, then the question arises whether $\theta_{23}>(D$ positive) or $\theta_{23}<\pi / 4$ ( $D$ negative). This leads to an additional two-fold degeneracy in the measurement of the mixing angle $\theta_{13}$ and the CP phase $\delta$ in LBL experiments. This ambiguity is generally regarded as the most difficult to resolve. As discussed before, the SK $\nu_{\mu} / \bar{\nu}_{\mu}$ data is insensitive to the sign of $D$. The measurements from the disappearance channel in the forthcoming LBL experiments are also going to be insensitive to the sign of $D$. However, matter effects in $P_{\mu \mu}$ open up a new possibility for an ICAL-like detector - sensitivity to the octant of $\theta_{23}$.

Fig. 6] shows the results of our statistical analysis based on simulated data from 1 MtonY exposure in an ICAL-like atmospheric $\nu_{\mu} / \bar{\nu}_{\mu}$ experiment. The left-hand panel gives the $\chi^{2}$ as a function of $\sin ^{2} \theta_{23}$, for a "data" simulated by assuming that $\sin ^{2} \theta_{23}$ (true) $=0.42$. For every 


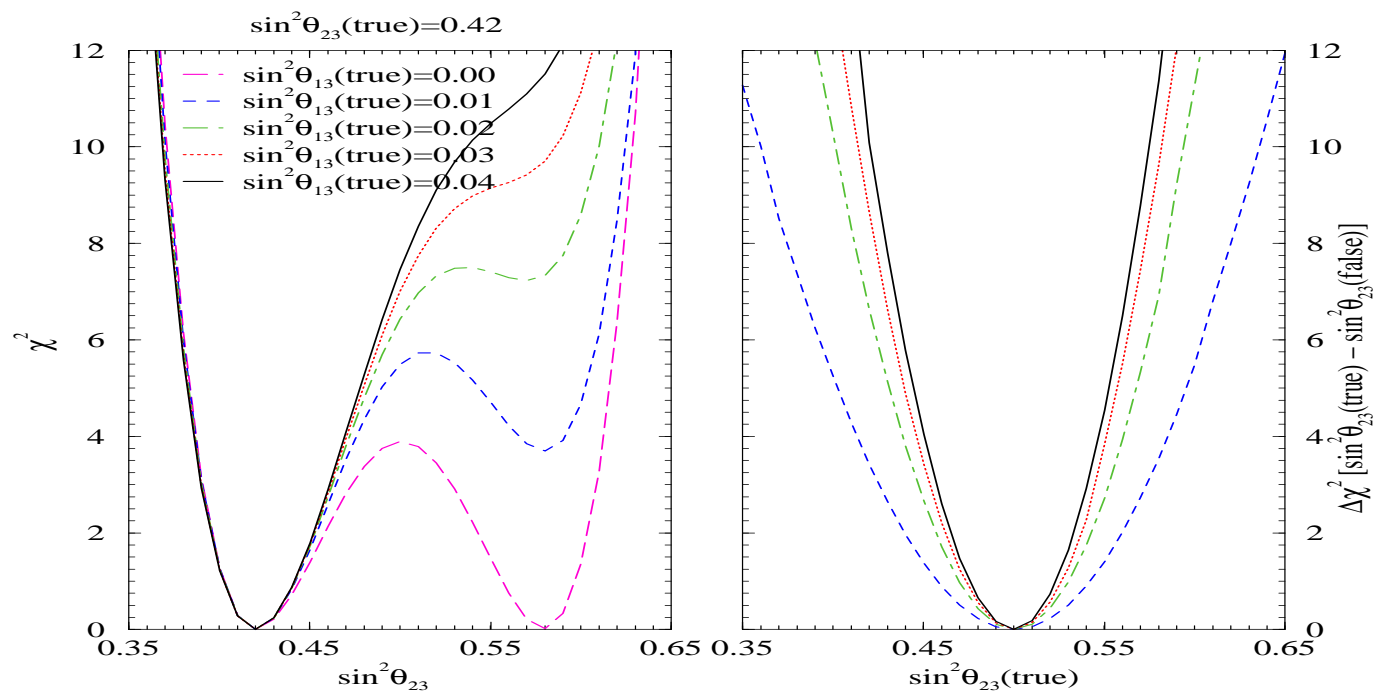

FIG. 6: Plot showing the octant sensitivity of ICAL. Left-hand panel shows $\chi^{2}$ as a function of $\sin ^{2} \theta_{23}$ for $\sin ^{2} \theta_{23}$ (true) $=0.42$. Right-hand panel gives the $\Delta \chi^{2}$ as a function of $\sin ^{2} \theta_{23}(\operatorname{true})$.

nonmaximal $\sin ^{2} \theta_{23}$ (true), there exists a $\sin ^{2} \theta_{23}$ (false) which is given by $\sin ^{2} \theta_{23}$ (false) $=$ $1-\sin ^{2} \theta_{23}$ (true) on the other side of $\pi / 4$. For a vanishing $\sin ^{2} \theta_{13}$ (true) there are no matter effects and the $\chi^{2}$ corresponding to both the true and false values of $\sin ^{2} \theta_{23}$ are the same. Hence they are allowed at the same C.L. and one fails to fix the octant of $\theta_{23}$ in this case. However, for $\sin ^{2} \theta_{13}$ (true) $\neq 0$, matter effects bring in an octant sensitivity and a false $\sin ^{2} \theta_{23}$ solution can be ruled out, provided $D$ (true) is not too close to zero.

In order to obtain the limiting value of $\sin ^{2} \theta_{23}$ (true) which could still allow for the determination of $\operatorname{sgn}(D)$ we define

$$
\left.\Delta \chi^{2} \equiv \chi^{2}\left(\sin ^{2} \theta_{23}(\text { true }), \sin ^{2} \theta_{13}(\text { true }), \text { others (true }\right)\right)-\chi^{2}\left(\sin ^{2} \theta_{23}(\text { false }), \sin ^{2} \theta_{13}, \text { others }\right),(2)
$$

with 'others' comprising $\Delta m_{31}^{2}, \Delta m_{21}^{2}, \sin ^{2} \theta_{12}$ and $\delta$. These, along with $\sin ^{2} \theta_{13}$, are allowed to vary freely in the fit. The right-hand panel of Fig. 6] shows $\Delta \chi^{2}$ as a function of $\sin ^{2} \theta_{23}$ (true) for different values of $\sin ^{2} \theta_{13}$ (true). The range of $\sin ^{2} \theta_{23}$ (true), for which $\sin ^{2} \theta_{23}$ (false) can be ruled out is visible from the figure. In particular, $\sin ^{2} \theta_{23}$ (false) should be excludable at the $3 \sigma$ level from 1 MtonY of ICAL exposure for $\sin ^{2} \theta_{23}$ (true) $<0.424$ or $>$ 0.570 for $\sin ^{2} \theta_{13}($ true $)=0.04$. 

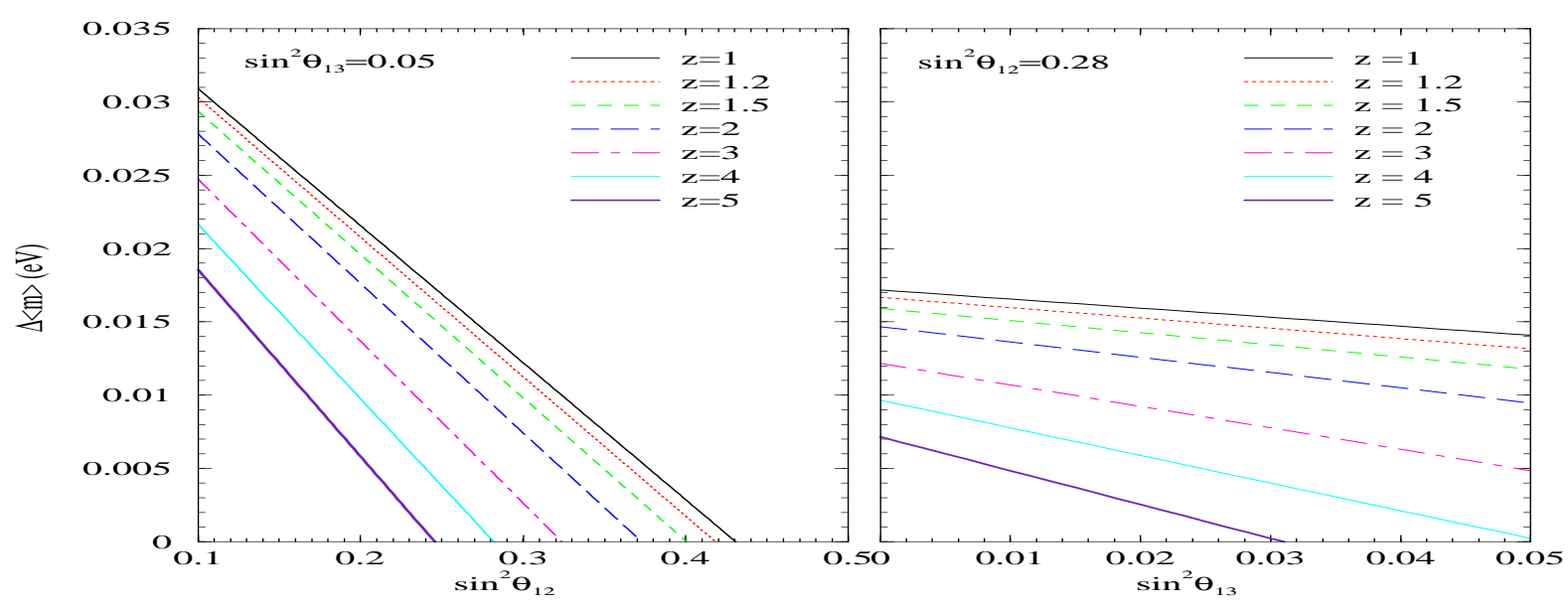

FIG. 7: The difference between the minimal value of $\langle m\rangle$ for IH and the maximal value of $\langle m\rangle$ for $\mathrm{NH}$ for different $z$, as a function of $\sin ^{2} \theta_{12}$ (left-hand panel) and $\sin ^{2} \theta_{13}$ (right-hand panel).

\section{NEUTRINO MASS HIERARCHY FROM FUTURE $0 \nu \beta \beta$ EXPERIMENTS}

Arguably the most fundamental question, whether neutrinos are Dirac or Majorana particles, remains to be answered. If neutrinos are Majorana particles, in principle it should be possible to observe the process $(A, Z) \rightarrow(A, Z-2)+2 e^{-}$, in which we have two $\beta$ particles in the final state and no neutrinos to accompany them - the process called neutrinoless double beta decay $(0 \nu \beta \beta)$. The effective mass which will be extracted or bounded in a $0 \nu \beta \beta$ experiment is given by the following coherent sum: $\langle m\rangle=\left|\sum_{i} m_{i} U_{e i}^{2}\right|$, where $m_{i}$ is the mass of the $i^{t h}$ neutrino mass state, the sum is over all the light neutrino mass states and $U_{e i}$ are the matrix elements of the neutrino mixing matrix. This means that $\langle m\rangle$ depends on 7 out of 9 parameters contained in the neutrino mass matrix, not depending on only the Dirac phase $\delta$ and $\theta_{23}$. In particular, effective mass to be extracted from neutrinoless double beta decay depends crucially on the neutrino mass spectrum. There have a been a large number of papers written on the implications of a future (non)measurement of $\langle m\rangle$ (see e.g. [19]). The best current limit on the effective mass is given by the Heidelberg-Moscow collaboration [20] $\langle m\rangle \leq 0.35 z \mathrm{eV}$, where $z(=\mathcal{O}(1))$ indicates that there is an uncertainty in the value of the nuclear matrix elements (NME) involved in the $0 \nu \beta \beta$ process. Several new experiments are currently running, under construction or in the planing phase [21]. Thus we expect $\langle m\rangle$ to be probed down to $\simeq 0.04 \mathrm{eV}$ and it would be pertinent to ask if such a measurement could help us learn about the neutrino mass hierarchy. 
For the NH scheme, for $m_{1} \ll m_{2} \ll m_{3}$ and assuming that $m_{1}$ can be neglected, we have

$$
\langle m\rangle^{\mathrm{NH}} \simeq\left|\sqrt{\Delta m_{21}^{2}} \sin ^{2} \theta_{12} \sin ^{2} \theta_{13}+\sqrt{\Delta m_{31}^{2}} \sin ^{2} \theta_{13} e^{2 i(\beta-\alpha)}\right| .
$$

For the IH scheme, assuming that $m_{3} \ll m_{1}<m_{2}$ and neglecting $m_{3}$, we have

$$
\langle m\rangle^{\mathrm{HH}} \simeq \sqrt{\left|\Delta m_{31}^{2}\right|} \sin ^{2} \theta_{13} \sqrt{1-\sin ^{2} 2 \theta_{12} \sin ^{2} \alpha} .
$$

Any future positive signal for $0 \nu \beta \beta$ will be able to distinguish the IH scheme from the $\mathrm{NH}$ scheme if the difference between the predicted values for $\langle m\rangle$ for the IH scheme and the $\mathrm{NH}$ scheme is larger than the error in the measured value of $\langle m\rangle$. Among the most important errors involved is the one coming from the uncertainty involved in the value of the nuclear matrix elements. In Fig. 7 we show the difference in the predicted values of $\langle m\rangle_{\max }^{\mathrm{NH}}$ and $\langle m\rangle_{\min }^{\mathrm{IH}}{ }^{2}$, taking into account the error in the nuclear matrix elements. This error is incorporated through the parameter $z$, which gives the factor by which the nuclear matrix elements is uncertain. See 22] for the details. It was argued 22] that for a given mass hierarchy, the uncertainty in the prediction of $\langle m\rangle$ coming from the uncertainty in the allowed values of $\Delta m_{31}^{2}$ and $\Delta m_{21}^{2}$ can be neglected, since these parameters are expected to be measured with very high accuracy in the immediate future. Therefore the major uncertainty in $\langle m\rangle$ would come from the still uncertain range for the values of $\sin ^{2} \theta_{12}$ and $\sin ^{2} \theta_{13}$. The Fig. 7 shows the impact of the uncertainty in the values of $\sin ^{2} \theta_{12}$ and $\sin ^{2} \theta_{13}$ on the sensitivity of the future $0 \nu \beta \beta$ experiments to the neutrino mass hierarchy. We see from the Fig. 7 that for $\sin ^{2} \theta_{13}$ close to its current limit and assuming $z=2$ and $\sin ^{2} \theta_{12}=0.3$, we have $\Delta\langle m\rangle \simeq 0.01 \mathrm{eV}$ and it should be possible to determine the neutrino hierarchy if the experimental uncertainty in $\langle m\rangle$ is less than $0.01 \mathrm{eV}$. The chances of determining the hierarchy is largest when $\sin ^{2} \theta_{13}=0$. More importantly, we note that while the dependence on $\sin ^{2} \theta_{13}$ is weak, the sensitivity of the $0 \nu \beta \beta$ experiments to the hierarchy is strongly dependent on $\sin ^{2} \theta_{12}$. Therefore the uncertainty on $\sin ^{2} \theta_{12}$ should be reduced well enough to get the neutrino mass hierarchy using $0 \nu \beta \beta$ experiments.

So far we assumed that the lightest neutrino mass was close to zero. If the lightest neutrino had a mass $m_{0} \gtrsim 0.01 \mathrm{eV}$, it would not be possible to distinguish between the $\mathrm{NH}$

${ }^{2}$ Since $\langle m\rangle^{\mathrm{NH}}$ and $\langle m\rangle^{\mathrm{NH}}$ depend on the parameters of the neutrino mass matrix, they have a range of allowed values. $\langle m\rangle_{\max }^{\mathrm{NH}}$ and $\langle m\rangle_{\min }^{\mathrm{IH}}$ are the largest and smallest predicted values for $\langle m\rangle$ in the $\mathrm{NH}$ and IH scheme respectively. 
and IH schemes using $0 \nu \beta \beta$ measurements. However, we could still use $0 \nu \beta \beta$ to put limit on the absolute neutrino mass scale. For quasi-degenerate (QD) mass spectrum with a common mass scale $m_{0}$, the limit on the neutrino mass reads [22]

$$
m_{0} \leq z\langle m\rangle_{\min }^{\exp } \frac{1+\tan ^{2} \theta_{12}}{1-\tan ^{2} \theta_{12}-2\left|U_{e 3}\right|^{2}} \equiv z\langle m\rangle_{\min }^{\exp } f\left(\theta_{12}, \theta_{13}\right) .
$$

Currently the uncertainty on $f\left(\theta_{12}, \theta_{13}\right)$ is around $50 \%, 1.9<f\left(\theta_{12}, \theta_{13}\right)<5.6$. It is expected to reduce to $\sim 21 \%(\sim 9) \%$ at $3 \sigma$ if a low energy $p p$ solar neutrino experiment (reactor experiment at the SPMIN) would be built. The uncertainty depends only little on the value of $\theta_{13}$. From the current limit on the effective mass, $\langle m\rangle \leq 0.35 z \mathrm{eV}$, with the accepted value of $z \simeq 3$ and our current knowledge of $f\left(\theta_{12}, \theta_{13}\right)$, we can set a limit on $m_{0}$ of $5.6 \mathrm{eV}$, clearly weaker than the limit from tritium beta decay experiments. However, if $f\left(\theta_{12}, \theta_{13}\right)$ was known with an uncertainty of $20 \%$, say $2.7<f\left(\theta_{12}, \theta_{13}\right)<4.0$, then for $z\langle m\rangle_{\min }^{\exp }=0.1$ $\mathrm{eV}$ we could set the limits $0.3 \mathrm{eV} \lesssim m_{0} \lesssim 0.4 \mathrm{eV}$. Of course, if we have no signal for $0 \nu \beta \beta$, but just an upper limit on $z\langle m\rangle_{\min }$, we have no longer an allowed range on $m_{0}$, but an upper limit corresponding to the largest value in the range. From the examples given above, one can note that for the QD mass spectrum, a measurement or a better constraint on $\langle m\rangle$ will lead to a stronger limit on the absolute neutrino mass scale compared to the current limit from direct kinematical searches.

\section{CONCLUSIONS}

A comparative study of the next generation solar neutrino experiments reveals that while $\Delta m_{21}^{2}$ can be measured well in the KamLAND experiment, to measure $\sin ^{2} \theta_{12}$ with a similar accuracy one would need a SPMIN reactor experiment with baseline of $60-70 \mathrm{~km}$. The forthcoming LBL experiments are expected to give very good limits on $\Delta m_{31}^{2}$ and $\sin ^{2} 2 \theta_{23}$. Comparable limits can be achieved using atmospheric neutrino data in large magnetized iron detectors like ICAL. The maximality of $\theta_{23}$ can be tested and the octant of $\theta_{23}$ can be determined most efficiently using matter effects in $P_{\mu \mu}$. Neutrino mass hieararchy could be determined in either LBL experiments or in atmospheric $\nu_{\mu} / \bar{\nu}_{\mu}$ experiments if $\sin ^{2} \theta_{13}$ (true) is large. In $\sin ^{2} \theta_{13}=0$, one could still see the hierarchy through $0 \nu \beta \beta$. We probed the conditions needed for it and pointed out that $\sin ^{2} \theta_{12}$ should be determined very accurately to use a future measurement of $\langle m\rangle$ to determine the neutrino mass hierarchy. 
I wish to thank my collaborators, A. Bandyopadhyay, S. Goswami, S. T. Petcov, W. Rodejohann, D. P. Roy and P. Roy. It is also my pleasure to thank the organisers of NANP'05.

[1] Y. Ashie et al., Phys. Rev. D 71, 112005 (2005); E. Aliu et al., Phys. Rev. Lett. 94, 081802 (2005).

[2] T. Araki et al., Phys. Rev. Lett. 94, 081801 (2005); B. Aharmim et al., arXiv:nucl-ex/0502021; S. Fukuda et al., Phys. Lett. B 539, 179 (2002); B. T. Cleveland et al., Astrophys. J. 496, 505 (1998); C. Cattadori, Talk at Neutrino 2004, Paris, France, June 14-19, 2004.

[3] A. Bandyopadhyay et al., Phys. Lett. B 608, 115 (2005); S. Goswami, A. Bandyopadhyay and S. Choubey, Nucl. Phys. Proc. Suppl. 143, 121 (2005).

[4] A. Bandyopadhyay, S. Choubey and S. Goswami, Phys. Rev. D 67, 113011 (2003).

[5] A. Bandyopadhyay et al., Phys. Lett. B 581, 62 (2004).

[6] Y. Suzuki, talk at Neutrino 2004, June 14-19 (2004), Paris, France.

[7] A. Bandyopadhyay et al., Phys. Rev. D 72, 033013 (2005).

[8] J. F. Beacom and M. R. Vagins, arXiv:hep-ph/0309300.

[9] S. Choubey and S. T. Petcov, Phys. Lett. B 594, 333 (2004).

[10] M. Maltoni, et al., New J. Phys. 6, 122 (2004), hep-ph/0405172 v7.

[11] P. Huber et al., Phys. Rev. D 70, 073014 (2004).

[12] M. C. Gonzalez-Garcia, M. Maltoni and A. Y. Smirnov, Phys. Rev. D 70, 093005 (2004).

[13] http://www.imsc.res.in/ ino/

[14] S. Choubey and P. Roy, in preparation.

[15] S. Antusch et al., Phys. Rev. D 70, 097302 (2004).

[16] S. Palomares-Ruiz and S. T. Petcov, Nucl. Phys. B 712, 392 (2005); D. Indumathi and M. V. N. Murthy, Phys. Rev. D 71, 013001 (2005); R. Gandhi et al., arXiv:hep-ph/0411252.

[17] S. Choubey and P. Roy, arXiv:hep-ph/0509197.

[18] S. Choubey and P. Roy, Phys. Rev. Lett. 93, 021803 (2004).

[19] S. T. Petcov, hep-ph/0504166 and references therein.

[20] H. V. Klapdor-Kleingrothaus et al., Eur. Phys. J. A 12, 147 (2001).

[21] C. Aalseth et al., hep-ph/0412300 and references therein.

[22] S. Choubey and W. Rodejohann, Phys. Rev. D 72, 033016 (2005). 